Historic, Archive Document

Do not assume content reflects current scientific knowledge, policies, or practices. 



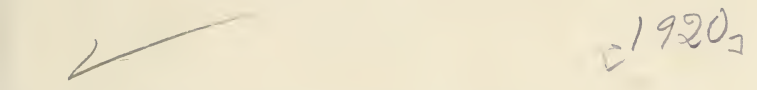

62.97 PRICE LIST

\section{RASMUSSEN'S FRUIT FARM}

N. A. RASmussen, Prop.
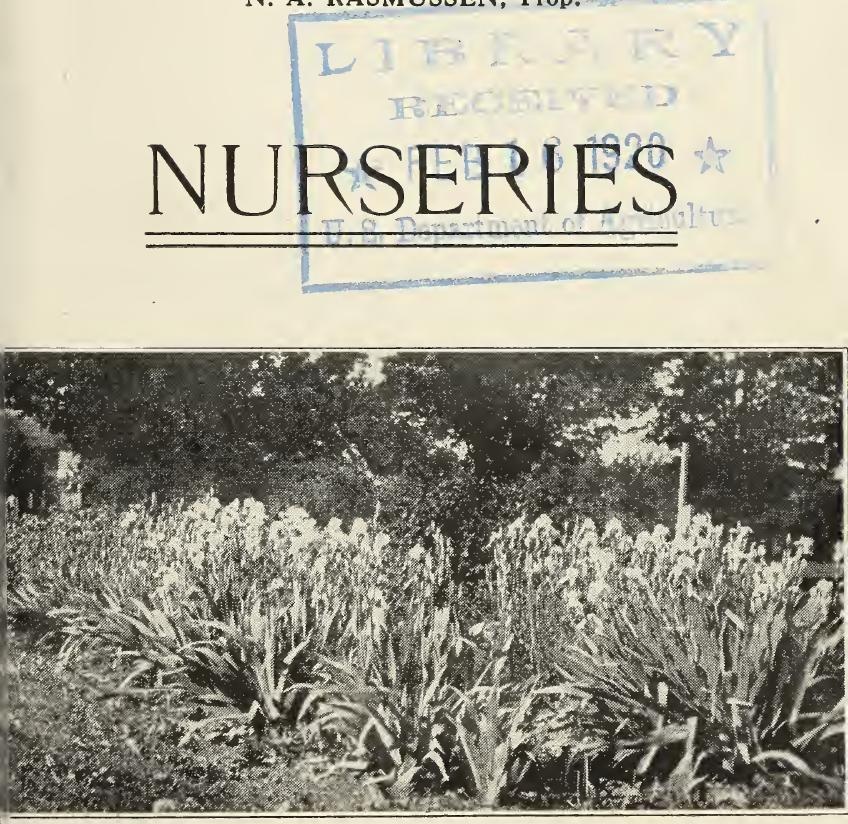

OSHKOSH, WISCONSIN

LICENSE NO. 173 


\section{ASPARAGUS}

\section{One and Two-Year-Old Roots}

Price per 25 $\$ .50$

Price per 100 1.50

Price per 1000 6.00

\section{RHUBARB}

\section{Large Strong Plants}

Price each $\$ .25$

Price two for .40

Price per dozen 2.00

\section{HORSERADISH}

Price per dozen ………………………………25c

\section{CHIVES}

Price per clump

$25 \mathrm{c}$

\section{SAGE}

Price each

$10 \mathrm{c}$

\section{HOT BED PLANTS IN SEASON}




\section{STRAWBERRIES}

We pack only good, strong plants and ship within 24 hours after digging. We grow the Warfield and SenDunlap exclusively, and recommend them for commercial and home use anywhere in the State of Wisconsin.

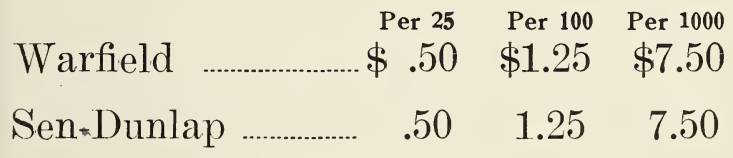

\section{Fall Bearing Varieties}
Americus
Per doz. Per 100
Superb
$\$ .50 \quad \$ 2.00$
Progressive
$.50 \quad 2.00$
.50
2.00

FOR LARGE QUANTITIES WRITE

FOR OUR SPECIAL PRICES 


\section{RASPBERRIES}

\section{Red}

$\begin{array}{lll}\text { Per doz. } & \text { Per } 100 & \text { Per } 1000\end{array}$

King ……..................... $.35 \quad \$ 1.50 \quad \$ 15.00$

LMarlborough $\quad$......... $\quad .35 \quad 1.50 \quad 15.00$

$\begin{array}{llll}\text { Cuthbert …................ } & .35 & 1.50 & 15.00\end{array}$

-St. Regis, Red Ever-

Bearing ………... $\quad .75 \quad 4.00$

Black
Greg's
50
Per 100
$\$ 2.50$
Cumberland
2.50

\section{CURRANTS}

\section{Perfection $\$ .60 \$ 6.00$}

Each Per doz.

GOOSEBERRIES

Industry

each $\$ 1.00$ 


\section{STANDARD APPLE TREES}

\section{Good Sized-Well Branched, 2 and 3 Years Old}

Fameuse (snow)

Each Perdoz. -Wealthy $1.00 \quad 10.00$ Yellow Transparent ......... $1.00 \quad 10.00$ Wolf River $1.00 \quad 10.00$

N. W. Greening. $1.00 \quad 10.00$ McIntosh $1.00 \quad 10.00$

Tolman Sweet $1.00 \quad 10.00$

Delicious $1.00 \quad 10.00$ Hyslop Crab Apple $1.00 \quad 10.00$

\section{CHERRY}

Early Richmond .each $\$ 1.00$ Montmorency each 1.00 May Duke (sweet) each 1.00

\section{PLUMS}

Surprise (native) each $\$ 1.00$

$\checkmark$ De Soto (native) each 1.00 Burbank (Japanese) each 1.00 


\section{HANSEN HYBRID PLUMS}

Sapa each $\$ 1.00$

Opata each 1.00

\section{FLOWERING SHRUBS}

Spirea Van Houttei .each $\$ .60$

Barberry Thunberg each .60

Purple Lilac each .25

Snow Berry White. each .60

\section{GERMAN IRIS}

\section{A Variety of Colors}

Light and dark blue, yellow, cream, lilac purple, etc.,

large clumps …_._...........each $\$ .25$

\section{TOMATO SEED}

The famous Buckstaff, pkt. 25c; oz. $\$ 1.25$ 


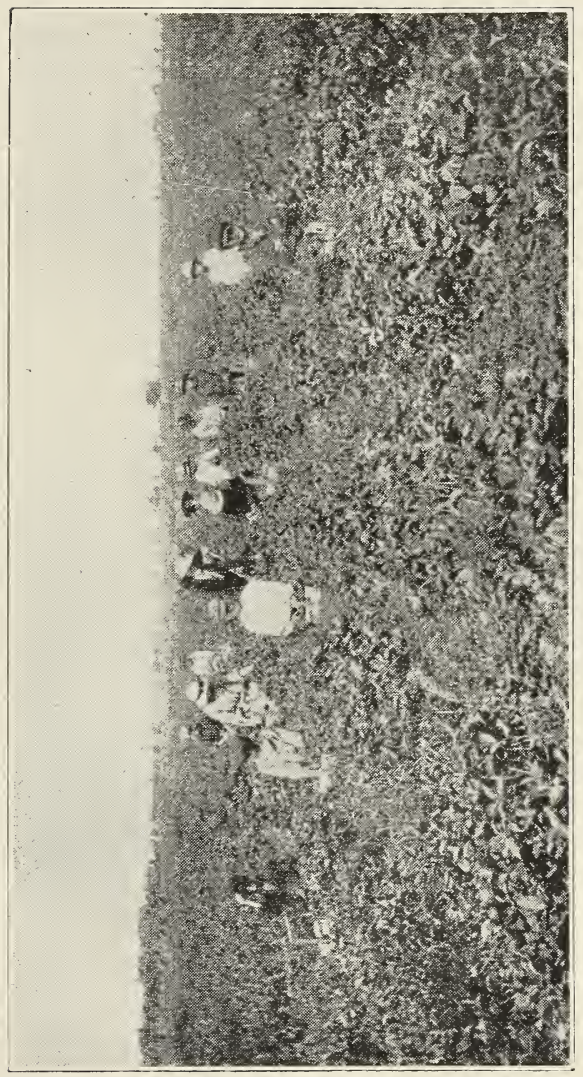




\section{Pure Bred}

Guernsey Cattle and

Single Comb Brown Leghorn Chickens

STOCK FOR SALE

Topsy May's Governor 52919

Heads Our Herd

Visitors always welcome at our Stables and Gardens. Oshkosh-Omro Interurban cars stop at our door. Leave Oshkosh station at 15 minutes past even hours daily. 